\title{
18. MODEL ATMOSPHERES \\ FOR HYDROGEN-DEFICIENT WHITE DWARFS
}

\author{
I. BUES
}

Institut für theoretische Physik und Sternwarte der Universität Kiel, Germany

\begin{abstract}
The determination of atmospheric parameters for non-DA white dwarfs is investigated with the computed helium-rich model atmospheres by Bues (1970). Only poor predictions are possible from UBV colors alone for DB and DC stars. From uvby colors a determination of effective temperature is possible within $1000 \mathrm{~K}$. Profiles of lines in different parts of the spectrum are necessary for better results.

A deficiency of metal abundances for the cooler non-DA stars is obtained.
\end{abstract}

For hydrogen-deficient white dwarfs the determination of atmospheric parameters is more uncertain than for DA-stars, since the different chemical composition is not specified in advance and the absorption coefficients of elements other than hydrogen are only approximately available.

As a first step in model computation for non-DA white dwarfs we therefore computed line-free flux-constant model atmospheres for 13 different helium-rich compositions (ratio $\mathrm{H}: \mathrm{He}$ varying from $1: 10$ to $1: 10^{6}$ ) with metal abundances varied, too, in the temperature range $11000-21000 \mathrm{~K}$ with $\log g=7$ and 8 (Bues 1970), now continued to $9000 \mathrm{~K}$. Besides $\mathrm{He}$ and $\mathrm{He}^{-}$metals must be considered for pressure and absorption. They determine pressure, stratification and flux in models with $T_{\text {eff }}<14000 \mathrm{~K}$ for a hydrogen deficiency of more than a factor of $10^{3}$.

The models were first compared with non-DA white dwarfs in the UBV two color diagram, where these stars occupy the region around the black body line between 8000 and $20000 \mathrm{~K}$ with the DB stars clustering near the position of a black body of $15000 \mathrm{~K}$. This position is reached for models with a ratio $\mathrm{H}: \mathrm{He} \leqslant 1: 10^{4}, 16000 \geqslant$ $\geqslant T_{\text {eff }} \geqslant 11000 \mathrm{~K}$. The cooler models form a sequence parallel to the black body line, the effective temperature being two thousand degrees lower than black bodies of equal color.

Up to this point the influence of lines on stratification and flux was neglected. For the structure of the models blanketing by HeI lines is less important than that of Balmer and Lyman lines for DA-stars because of the relative weakness of the lines. Metal lines would be more important if metals were present at solar abundances. For the UBV colors, however, blocking effects of both, helium and metal lines have to be taken into account for all three filters and thus a temperature determination for DB and DC stars from observed colors becomes difficult.

More suitable for helium-rich stars is the intermediate band system of Strömgren, uvby: the $\mathrm{u}$ filter is centered at the steepest absorption edge of $\mathrm{He}_{\mathrm{I}}$ in the near UV and affected by $\mathrm{HeI}$ lines for models hotter than $14000 \mathrm{~K}$, the $\mathrm{b}$ filter is influenced by the weak He I line at $4713 \AA$ (max. $W_{\lambda} \approx 8 \AA$ for $\left.T_{\text {eff }}=15500 \mathrm{~K}\right)$, whereas the $y$ filter covers a line-free region. Figure 1 shows the experimental data for $6 \mathrm{DB}$ and 


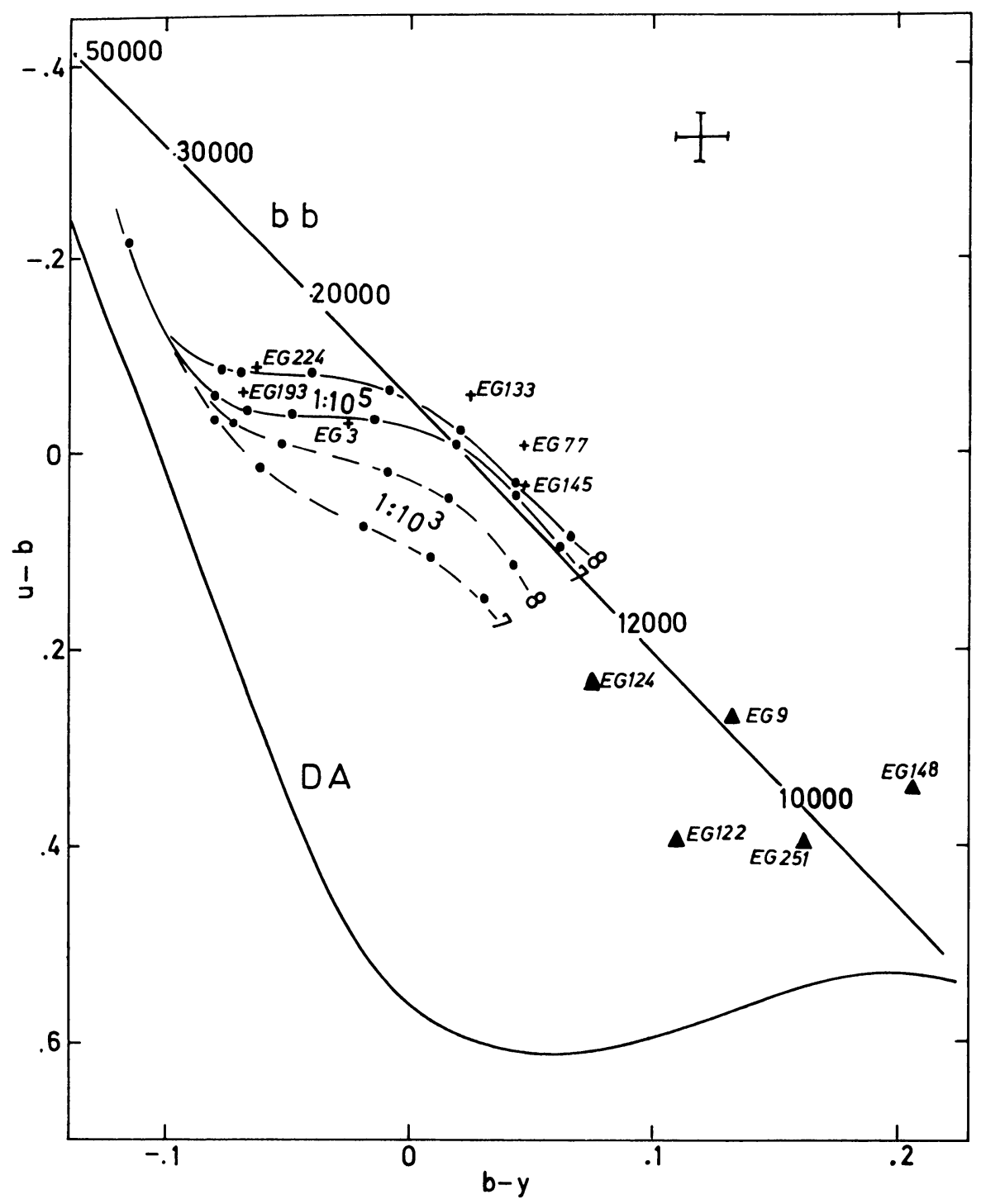

Fig. 1. Strömgren color diagram for white dwarfs. Observed colors for $\mathrm{DB}(+)$ and $\mathrm{DC}(\boldsymbol{\Delta})$ stars compared to models with normal metal abundances, $\log g=7$ and 8 . From right to left $T_{\text {eff }}=10000$, $11000,12000,13200,15500,18000,21000$ and $30000 \mathrm{~K}$ is marked by the dots for $\mathrm{H}: \mathrm{He}=1: 10^{5}$. For $\mathrm{H}: \mathrm{He}=1: 10^{3}$ models with $T_{\text {eff }}=10000$ and $21000 \mathrm{~K}$ are omitted.

5 DC stars, kindly provided by Graham (1970) and the computed colors for models with solar metal abundances by mass. The error bars indicate the assumed accuracy of measurements. Here we see a well defined sequence of DB stars which does not appear in the UBV two color diagram for the same stars. The observed sequence is reproduced by computed colors for a hydrogen abundance equal or smaller than $1: 10^{5}$. (The position does not change with further reductions of hydrogen.) 


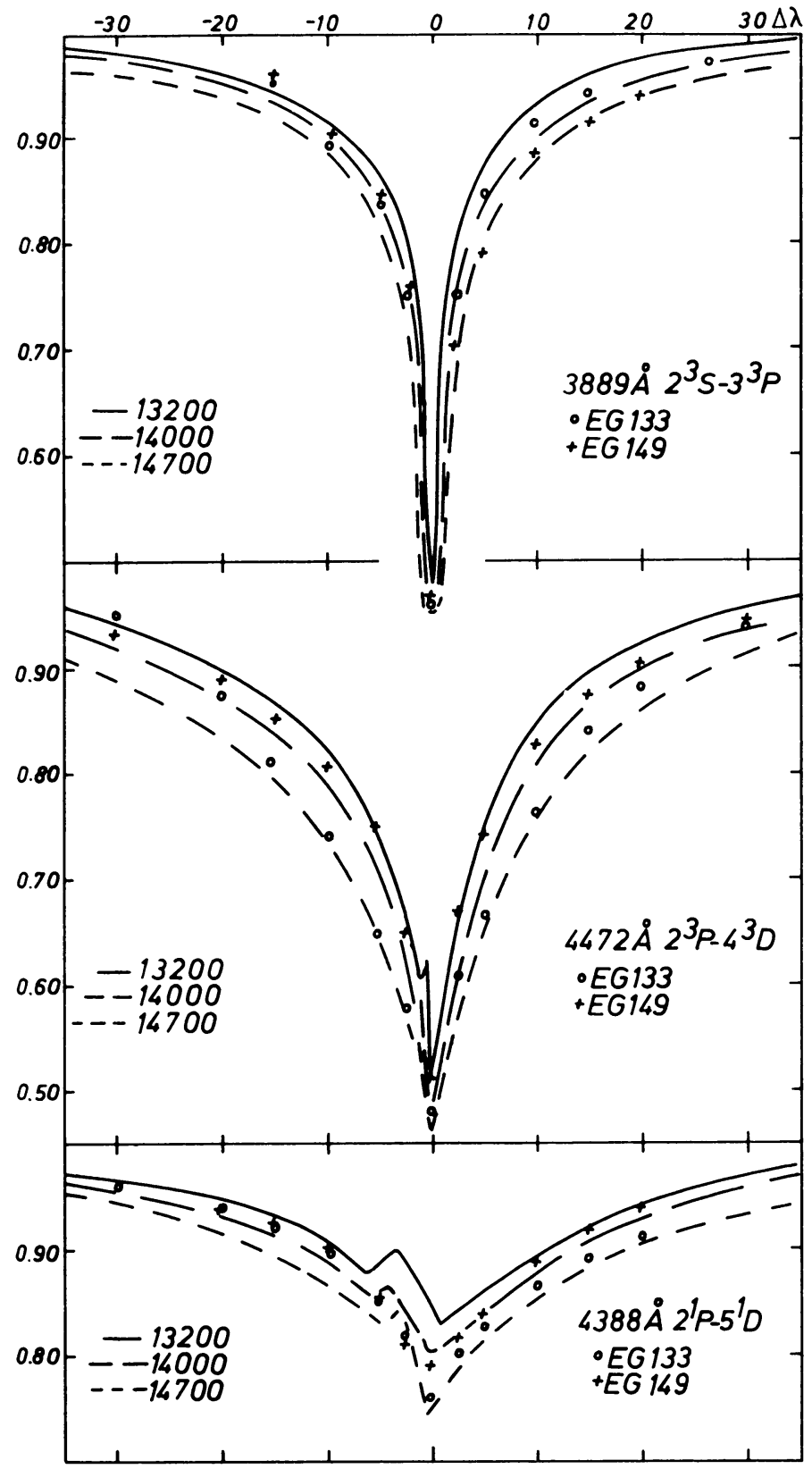

Fig. 2. Line profiles (in $\AA$ ) of $3 \mathrm{He}$ l lines for 3 models with composition $\mathrm{H}: \mathrm{He}=1: 10^{5}, Z=0.02$, $\log g=7$ compared with observations for EG 133 and EG 149. 
Some uncertainties arise for this system from the absolute calibration. The calibration constant obtained by Matsushima (1969) with fluxes of line-blanketed model atmospheres for A stars and ours, which was derived with the flux of $\delta$ Del analysed by Reimers (1969), differs by $0.02 \mathrm{mag}$ in $(u-b)$. The corresponding differences in $\log g$ are within the accuracy of computation and temperature determination is possible only within $1000 \mathrm{~K}$ for the cooler DB stars.

An improved determination of temperatures is possible by consideration of line profiles. Observed profiles are available for EG 133, EG 149 and EG 145 from Greenstein (1960). The application of broadening theories of Griem et al. (1962), Griem (1969) and Pfennig and Trefftz $(1965,1966)$ to $10 \mathrm{He}$ I lines yielded the observed line depths for models with $12000 \leqslant T_{\text {eff }} \leqslant 15500 \mathrm{~K}, \log g=7$. A significant difference in line shape (5-25 $\AA$ from the center) between $\log g=7$ and 8 exists for models with $T_{\text {eff }} \leqslant 14000 \mathrm{~K}$ only. A comparison of observed and computed profiles for EG 133 and EG 149 is shown in Figure 2 at normal metal abundances.

From relative intensities and shapes of lines in different spectral regions better agreement with observation is obtained if metal abundances are reduced by a factor of 100 . The results for these two stars are $T_{\text {eff }}=14700 \pm 500 \mathrm{~K}$ for EG 133 and $T_{\text {eff }}=14000 \pm 500 \mathrm{~K}$ for EG 149 , respectively.

An independent check on metal abundances is the calculation of strong metal lines, most important being $\mathrm{Ca}$ II $\mathrm{H}$ and $\mathrm{K}$ and $\mathrm{Mg}$ I $\lambda \lambda 3829,3832$ and $3838 \AA$. The equivalent width of the $\mathrm{H}$ and $\mathrm{K}$ blend for $T_{\text {eff }}=13200 \mathrm{~K}, \log g=8$ is $30 \AA$ ( $\max \approx 40 \AA$ for $T_{\text {eff }}=11000 \mathrm{~K}$ ) with a sharp line center (central depth $70 \%$ ) and halfwidths of the order of $10 \AA$. Thus it must be concluded that $\mathrm{Ca}$ is deficient by a factor of at least 100 for $\mathrm{DB}$ stars. For $\mathrm{Mg}, \mathrm{Si}, \mathrm{Fe}$ and $\mathrm{Ni}$ reduction factors of $\sim 10$ are necessary in this range of temperature. For lower temperatures, $T_{\text {eff }} \leqslant 11000 \mathrm{~K}$, the computed line strengths increase because of the relatively low $\mathrm{He}^{-}$absorption coefficient and a DC continuum would not appear for normal metal abundances, although the strongest He I line in the visible region, $\lambda 4472 \AA$, is below visibility. $\left(W_{\lambda} \leqslant 0.4 \AA\right.$ for $\left.T_{\text {eff }} \leqslant 10000 \mathrm{~K}\right)$

Lines of nitrogen and carbon are too weak to be visible in the spectra.

Since the $\lambda 4670$ stars evidently have carbon, we calculated the partial pressure of $\mathrm{C}_{2}$ and derived abundances relative to $\mathrm{C}_{\mathrm{I}}$ at each depth of models with $T_{\mathrm{eff}}=11000$ and $10000 \mathrm{~K}$. With $f$ values of Smith (1969) a strength of 0.1 and $0.5 \AA$, respectively, for the Swan-band was obtained, whereas the sum of the $6 C_{I}$ lines $\lambda \lambda 4768-4775 \AA$ resulted in a total equivalent width of 2 and $6 \AA$, respectively. This may indicate an effective temperature below $10000 \mathrm{~K}$ for the $\lambda 4670$ stars if they have equal composition, since the Swan-band is predominant in their spectra. Vice versa, for the DC stars, we must conclude that carbon is deficient, because the intensities of the $\mathrm{CI}_{\mathrm{I}}$ lines increase for lower effective temperatures and should be visible $\left(W_{\lambda}>6 \AA\right)$ for normal abundance.

\section{References}

Bues, I.: 1970, Astron. Astrophys. 7, 91.

Graham, J. A.: 1970, private communication. 
Greenstein, J. L.: 1960, Stars and Stellar Systems 6 (ed. by J. L. Greenstein), University of Chicago Press, Chicago, p. 676.

Griem, H. R.: 1968, Astrophys. J. 154, 1111.

Griem, H. R., Baranger, M., Kolb, A. C., and Oertel, G.: 1962, Phys. Rev. 125, 177.

Matsushima, S.: 1969, Astrophys. J. 158, 1137.

Pfennig, H. and Trefftz, E.: 1965, Instit. ber. MPI/PAE/Astro. Nr. 18, Munich.

Pfennig, H. and Trefftz, E.: 1966, Z. Naturforsch. 21a, 697.

Reimers, D.: 1969, Astron. Astrophys. 3, 94.

Smith, W. H.: 1969, Astrophys. J. 156, 791. 